\title{
Scanning Tunneling Spectroscopy of Cl Vacancies in NaCl Films: Strong Electron-Phonon Coupling in Double-Barrier Tunneling Junctions
}

\author{
Jascha Repp, ${ }_{1}^{1}$ Gerhard Meyer, ${ }^{1}$ Sami Paavilainen, ${ }^{2, *}$ Fredrik E. Olsson, ${ }^{2}$ and Mats Persson ${ }^{2}$ \\ ${ }^{1}$ IBM Research, Zurich Research Laboratory, 8803 Rüschlikon, Switzerland \\ ${ }^{2}$ Department of Applied Physics, Chalmers, 41296 Göteborg, Sweden
}

(Received 18 February 2005; published 21 November 2005)

\begin{abstract}
Broad Gaussian line shapes are observed in scanning tunneling spectroscopy of single, localized electronic states induced by $\mathrm{Cl}$ vacancies in ultrathin $\mathrm{NaCl}$ films on $\mathrm{Cu}$ surfaces. Using a simple inelastic resonance tunneling model, we show that the observed broad line shapes are caused by a strong coupling between the localized state and the optical phonons in the film. The parameters for the model are obtained from density functional calculations, in which the occupation of the vacancy state temporarily taking place in the experiment has also been accounted for.
\end{abstract}

DOI: 10.1103/PhysRevLett.95.225503

PACS numbers: 63.20.Mt, 68.37.Ef, 71.15.Mb, 73.20.Hb

The recent spectacular progress in carrying out scanning tunneling microscopy (STM) and spectroscopy (STS) of adsorbates on ultrathin, insulating films supported by metal surfaces has opened up a new fascinating field in atomicscale science [1-4]. This field is of key importance, for example, in the realization of any future atomic-scale electronics that will necessarily be based on nanostructures hosting confined electronic states, which are insulated from the environment by nanostructured, wide-band-gap materials. A fundamental issue is the degree of decoupling of localized electronic states by an insulating film. The well-known lifetime broadening - the dominant broadening mechanism for localized electronic states on metal surfaces - should be dramatically reduced by an insulating film. Therefore, it could be expected that the adsorbate and defect-induced states on insulators exhibit very small linewidths. However, recent STS experiments revealed the contrary $[5,6]$. This apparent contradiction triggered this study on the different broadening mechanisms and resulting line shapes and widths in STS.

An ideal model system for the study of tunneling through a localized electronic state decoupled by a polar insulator from the metal substrate and the associated line shape is provided by a single $\mathrm{Cl}$ vacancy in $\mathrm{NaCl}$ films on $\mathrm{Cu}$ surfaces. This atomic-scale defect in an otherwise defect-free environment is fairly simple, well defined, and stable. The intrinsic defects in bulk and at surfaces of $\mathrm{NaCl}$ have been studied both experimentally and theoretically in detail [7-9]. These studies have shown that a $\mathrm{Cl}$ vacancy, which is commonly referred to as a color or $F$ center, introduces a singly occupied defect state in the bulk band gap.

In this Letter, we show from STM and STS measurements that a single $\mathrm{Cl}$ vacancy in a $\mathrm{NaCl}$ film on a $\mathrm{Cu}$ surface introduces an unoccupied vacancy state (VS) with a broad Gaussian line shape in the $d I / d V$ spectra. Using a simple inelastic resonance tunneling model with parameters from density functional calculations, we show that the observed line shape is caused by a strong electron-phonon $(e-p h)$ coupling of the VS to the ionic lattice of the insulating film. This broadening mechanism should be a general phenomenon for defect and adsorbate states on polar, insulating films. For example, it provides an explanation of the recently observed, Gaussian broadening of the negative ion resonance in pentacene molecules adsorbed on $\mathrm{NaCl}$ films [5]. In addition, the positive charge of the vacancy is confirmed on the $\mathrm{Cu}(111)$ surface by the formation of interface-state localization (ISL) at the vacancy.

The experiments were carried out with a home-built low-temperature STM operated at $T=5 \mathrm{~K} . \mathrm{Cu}(111)$, $\mathrm{Cu}(100)$, and $\mathrm{Cu}(311)$ single-crystal samples were cleaned by several sputtering and annealing cycles. $\mathrm{NaCl}$ was evaporated thermally, keeping the sample temperature at about $300 \mathrm{~K}$, so that defect-free, (100)-terminated $\mathrm{NaCl}$ islands with few atomic layers were formed [1,10-12]. Bias voltages refer to the sample voltage with respect to the tip. $\mathrm{Cl}$ vacancies were formed with the STM tip using two different techniques [13].

To obtain a better physical insight into the nature of these vacancies, we have carried out density-functional theory (DFT) calculations of their geometry and electronic states. The calculations were based on a plane wave basis set and a projector augmented wave method [14,15], as implemented in the VASP code [16] [for details, see Ref. [17] ]. Because growth of the $\mathrm{NaCl}$ films on $\mathrm{Cu}(111)$ is incommensurate, the calculations were limited to films supported by $\mathrm{Cu}(100)$ and $\mathrm{Cu}(311)$ surfaces. STM images were simulated using the Tersoff-Hamann approximation [18] in which STM images correspond to topographic images of constant local density of sample states (LDOS) at the Fermi energy and at the position of the tip apex. Finally, parameters for the $e$-ph coupling model were calculated using a $\Delta$ SCF-like scheme [19] within DFT as described below.

In STM, $\mathrm{Cl}^{-}$ions are imaged as protrusions on $\mathrm{NaCl}$ films [20-22]. As illustrated in Fig. 1(a) for the $\mathrm{NaCl}$ 


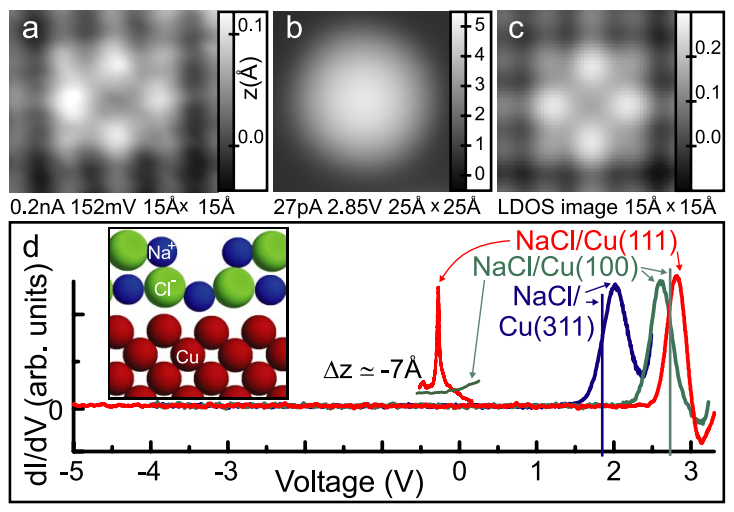

FIG. 1 (color online). Experimental (a), (b) and simulated STM images (c) of an individual $\mathrm{Cl}$ vacancy in the top layer of a bilayer of $\mathrm{NaCl}$ on $\mathrm{Cu}(111)$ (a), (b) and $\mathrm{Cu}(100)$ (c). Whereas the low-bias image (a), (c) shows a missing protrusion, the image at high bias voltage (b) shows a large protrusion of $4.5 \AA$ in height. (d) $d I / d V$ spectra at a $\mathrm{Cl}$ vacancy in a bilayer of $\mathrm{NaCl}$ on $\mathrm{Cu}(311), \mathrm{Cu}(100)$, and $\mathrm{Cu}(111)$. The calculated peak positions are shown as vertical lines for $\mathrm{NaCl}$ bilayer on $\mathrm{Cu}(311)$ and $\mathrm{Cu}(100)$. In the case of $\mathrm{NaCl} / \mathrm{Cu}(111)$, there is an additional peak at $-0.26 \mathrm{~V}$ that can be resolved only when taking a spectrum with the tip being about $\Delta z \simeq-7 \AA$ closer to the surface. In the inset a sphere model of the vacancy geometry is shown.

bilayer on $\mathrm{Cu}(111)$, the $\mathrm{Cl}$ vacancies are imaged as missing protrusions accompanied by an increase in the corrugation of the adjacent $\mathrm{Cl}$ ions [23]. The assignment of the created defects to single $\mathrm{Cl}$ vacancies is verified by comparing the simulated and observed STM images in Fig. 1. The enhanced corrugation of the adjacent protrusions is caused by the outward relaxation of the $\mathrm{Cl}^{-}$ions. As shown by the $d I / d V$ spectra in Fig. $1(\mathrm{~d})$, the $\mathrm{Cl}$ vacancy in a $\mathrm{NaCl}$ bilayer on $\mathrm{Cu}(311), \mathrm{Cu}(100)$, and $\mathrm{Cu}(111)$ induces an unoccupied VS at 2.03, 2.57, and $2.83 \mathrm{~V}$, respectively. The peak positions follow roughly the differences in work functions for the clean $\mathrm{Cu}$ facets. The state is localized at the vacancy site, and for voltages exceeding the VS energy the tunneling is dramatically enhanced by 4 orders of magnitude compared to tunneling through the defectfree bilayer. This is revealed by the large protrusion of about $4.5 \AA$ in height in the STM image [Fig. 1(b)].

In the case of a double-barrier tunneling junction, one needs to correct for the voltage drop across the insulating film to obtain the energy of the VS in the absence of an applied electric field. The electric field and thus the voltage drop depend on the tip-surface distance $z+\Delta z$. As shown in the inset of Fig. 2 for the $\mathrm{NaCl}$ bilayer on $\mathrm{Cu}(111)$, we find that the peak position shifts only slightly with $\Delta z$. Using a simple one-dimensional (1D) capacitor model [4], we estimate the VS energy in the absence of an external field to be $E_{\mathrm{VS}}=2.77 \pm 0.02 \mathrm{eV}$. Thus, the field induced shift is only a few percent of the bias voltage applied.

We experimentally determined the charge state of the vacancy as described in the following. As shown by the

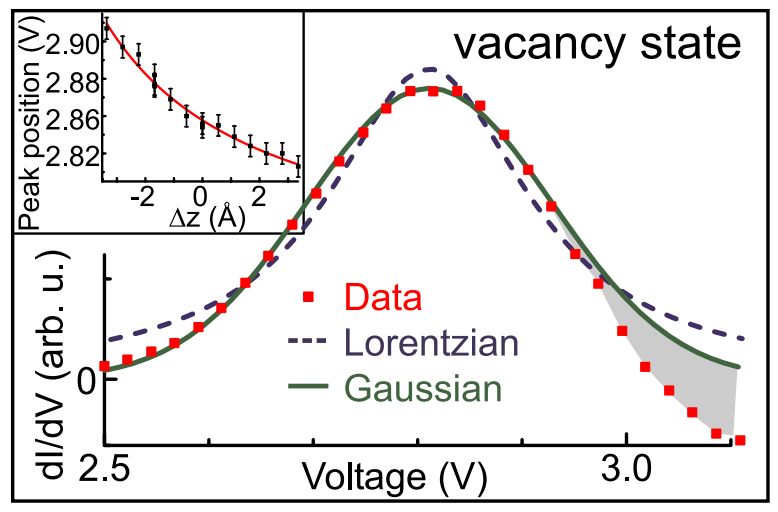

FIG. 2 (color online). $\quad d I / d V$ spectrum of the VS in a bilayer of $\mathrm{NaCl}$ on $\mathrm{Cu}(111)$ fitted to Lorentzian and Gaussian line shapes. The slight deviation from the Gaussian (shaded area) is in agreement with the tunneling barrier being modified by the applied voltage [33]. Inset: VS peak position for varying tipsurface distances. The best fit to a $1 \mathrm{D}$ capacitor model [4] is indicated by a solid line. $\Delta z=0$ refers to a current of $1.5 \mathrm{pA}$.

STM image in the inset of Fig. 3, the $\mathrm{Cl}$ vacancy efficiently scatters the Shockley-like interface-state electrons [11] localized in the interface of the $\mathrm{NaCl}$ bilayer and $\mathrm{Cu}(111)$. In addition, the defect-induced potential supports an ISL [24] showing up as a peak at $-0.26 \mathrm{~V}$ in the $d I / d V$ spectrum [Figs. 1(d) and 3]. This state is split off from the interface-state band just below the band bottom at $-0.22 \mathrm{~V}$ [11]. This assignment is supported by the absence of such a peak in the $d I / d V$ spectra for a $\mathrm{Cl}$ vacancy in the $\mathrm{NaCl}$ bilayers on $\mathrm{Cu}(311)$ and $\mathrm{Cu}(100)$, for which no interface-state band is present near the Fermi energy. In general, an ISL is expected to exist on insulating films on $\mathrm{Cu}(111)$ whenever the interaction of a two-dimensional,

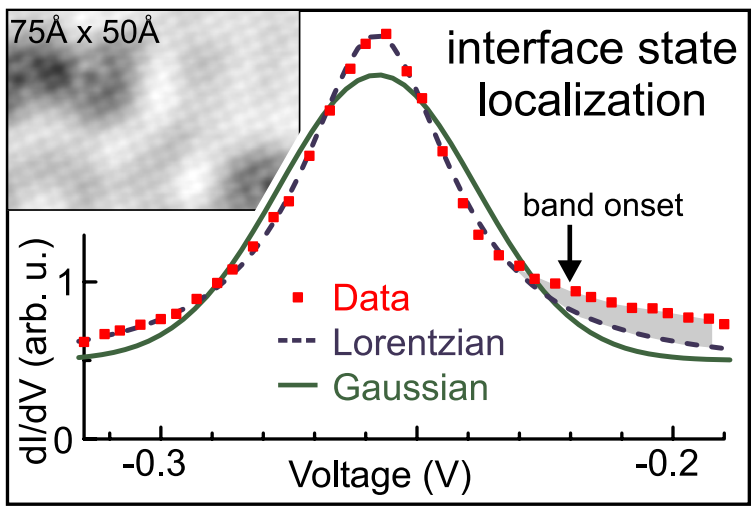

FIG. 3 (color online). $\quad d I / d V$ spectrum of the ISL in a bilayer of $\mathrm{NaCl}$ on $\mathrm{Cu}(111)$ fitted to Lorentzian and Gaussian line shapes. The slight deviation from the Lorentzian (shaded area) is due to the remnants of the interface-state band. For comparison, the position of the onset of the interface-state band for the defect-free surface is indicated by an arrow. Inset: STM image acquired at $V=58 \mathrm{mV}$ showing the scattering of the interface state at the vacancies. 
free-electron-like interface band with a defect is attractive [24]. In the case of the $\mathrm{Cl}$ vacancy such an attractive interaction is consistent with the vacancy being positively charged. For a comparison, a negatively charged defect, such as an $\mathrm{Au}^{-}$ion [1], is a strong scatterer of interfacestate electrons, but does not induce any ISL, whereas a neutral defect, such as an Au adatom, neither scatters the interface-state electrons nor induces an ISL. Thus, observation of the interface-state scattering and ISL provides information about the charge state of the defect. The interpretation that the vacancy is positively charged is corroborated by the calculated charge density.

The two localized states supported by a $\mathrm{Cl}$ vacancy in a $\mathrm{NaCl}$ bilayer on $\mathrm{Cu}(111)$ have different and characteristic line shapes in the $d I / d V$ spectra. As can be clearly seen in the Figs. 2 and 3, the VS peak is best fitted by a Gaussian, whereas the ISL peak is best fitted by a Lorentzian [25]. The corresponding full width at half maximum (FWHM) is $0.27 \mathrm{~V}$ for the VS and only $0.035 \mathrm{~V}$ for the ISL in the $\mathrm{NaCl}$ bilayer on $\mathrm{Cu}(111)$. The $d I / d V$ spectrum for the VS in the $\mathrm{NaCl}$ trilayer on $\mathrm{Cu}(111)$ also exhibits a Gaussian line shape with the same width as that for the bilayer and centered at $2.70 \mathrm{eV}$ for a tunneling current of $20 \mathrm{pA}$.

Interestingly, the peak of the ISL shows a much smaller width than for the VS despite the much larger spatial overlap of the ISL with the metal states. The Lorentzian line shape of the ISL corresponds to the well-known lifetime broadening which can arise from several mechanisms [26]. In the case of the VS, the Gaussian line shape and the same FWHM values for the bilayer and trilayer cannot be easily reconciled with a broadening mechanism involving a coupling to the metal states.

Instead, we show that the broad Gaussian line shape of the VS in the $d I / d V$ spectra is a result of strong $e$-ph coupling in the tunneling through the VS. The argument is based on a simple inelastic resonance tunneling model in a double-barrier junction with linear coupling to phonon modes $[27,28]$. In this model for a single phonon mode with energy $\hbar \omega$, the line shape is given by a Poisson distributed set of peaks separated by $\hbar \omega$ and centered at the (vertical) electron attachment energy $\Delta E$ (Fig. 4). In the limit of strong $e$-ph coupling, the envelope of the vibronic peaks becomes a Gaussian with a FWHM of $\Delta=$ $\sqrt{8 \ln 2 S} \hbar \omega$ where the strength of the $e$-ph coupling appears through the Huang-Rhys factor $S$. This factor can be expressed in terms of the relaxation energy $E_{\text {rel }}$ (Fig. 4) of the phonon mode when the VS is occupied as $S=E_{\text {rel }} / \hbar \omega$. Note that the width $\Delta$ is the same as the width of the Gaussian distribution of the electron attachment energies to the VS caused by the zero-point fluctuations of the phonon mode (Fig. 4).

The above model requires values of three parameters to describe the peak position and width: a characteristic vibrational energy $\hbar \omega$, the excitation energy $\Delta E$ that is needed to attach an extra electron to the VS for a fixed

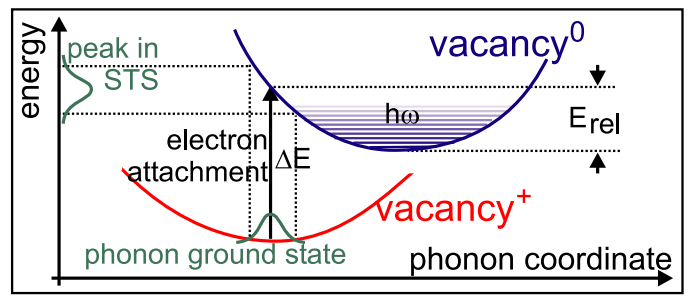

FIG. 4 (color online). Illustration of the broadening mechanism.

ionic geometry, and the ionic relaxation energy $E_{\text {rel }}$. As a value for $\hbar \omega$, we used the longitudinal optical (LO) phonon energy of bulk NaCl, $\hbar \omega=32 \mathrm{meV}$ [29], since this mode provides the strongest coupling to an external charge.

$\Delta E$ and $E_{\text {rel }}$ were calculated using the following $\Delta$ SCF-like scheme within DFT [19]. First, the calculated ground state electronic structure revealed that the VS was described by a single, unoccupied Kohn-Sham (KS) state localized at the vacancy site [30]. Note that this result supports our claim that the observed broadening of the VS is not caused by the coupling of the VS to the metal states through the film [31]. Next, this KS state was forced to be occupied by a single electron while keeping the whole system neutral by adjusting the Fermi level. $\Delta E$ was then calculated as the total energy difference between this excited state (occupied VS) in the ground state geometry and the ground state (unoccupied VS): $\Delta E=1.85$ and $2.73 \mathrm{eV}$ for the $\mathrm{VS}$ in the $\mathrm{NaCl}$ bilayer on $\mathrm{Cu}(311)$ and $\mathrm{Cu}(100)$ surfaces, respectively. Finally for $\mathrm{Cu}(100), E_{\text {rel }}$ was obtained as the gain in total energy by a full structural optimization while keeping the VS occupied: $E_{\text {rel }}=$ $0.96 \mathrm{eV}$.

As shown in Fig. 1(d), the calculated values for $\Delta E$ are in very good agreement with the observed VS peak positions. We find a strong $e$-ph coupling, $S=30$, for the vacancy in a $\mathrm{NaCl}$ bilayer on $\mathrm{Cu}(100)$, being almost as strong as for a $\mathrm{Cl}$ vacancy state in bulk $\mathrm{NaCl}[S=42$ [32] ]. The resulting estimate for the width, $\Delta=0.41 \mathrm{eV}$, accounts well for the observed FWHM of $0.32 \mathrm{~V}$ for the VS state of the corresponding system. Note that the discrete vibronic structure of the line shape in the model should be blurred in the spectra primarily by a vibronic contribution from a band of phonon modes.

In contrast to the VS, there is no significant broadening of the ISL due to the coupling to the optical phonons in the $\mathrm{NaCl}$ film. This can be understood qualitatively from the fact that the ISL wave function is similar to the Shockley interface-state wave function [11], and thus, is efficiently screened by the metal electrons.

On the other hand, any localized state that is not efficiently screened near a polar insulator is affected by the strong $e$-ph coupling as described for the VS. For example, this mechanism provides an explanation of the observation in recent STS measurements of a Gaussian-shaped peak from the negative ion resonance in pentacene on $\mathrm{NaCl}$ 
films with a FWHM of $0.30 \mathrm{eV}$, which is way beyond the expected lifetime broadening [5]. As in the present case, the FWHM did not change from two to three layers of $\mathrm{NaCl}$ thickness. Strong coupling to phonons should also explain the broad peaks observed in $d I / d V$ spectra of $\mathrm{Pd}$ adatom states on the ultrathin polar insulating $\mathrm{Al}_{2} \mathrm{O}_{3}$ film on $\mathrm{NiAl}[6]$.

In conclusion, the observed broad line shapes in STS from defects and adsorbate-induced electronic states on ultrathin, polar films do not indicate that these films are leaky but should in general be an effect of the strong coupling of localized electronic states to optical phonons in the film. This finding comes from STM and STS studies of single $\mathrm{Cl}$ vacancies in and adsorbed pentacene molecules on ultrathin $\mathrm{NaCl}$ films on $\mathrm{Cu}$ surfaces. The $\mathrm{Cl}$ vacancies in a $\mathrm{NaCl}$ film exhibit an unoccupied vacancy state with a broad Gaussian line shape in the STS, which is explained in a simple inelastic resonance tunneling model with parameters from density functional calculations. Finally, useful information about the charge state of a defect or adsorbate on an insulating film on $\mathrm{Cu}(111)$ can be obtained from STM imaging of interface-state scattering combined with STS measurement of any induced interface-state localization.

Valuable comments by Rolf Allenspach, financial support by the EU projects "AMMIST," "CHIC," and "NANOMAN," Academy of Finland, Swedish Research Council (VR), Swedish Strategic Foundation (SSF) through materials consortium "ATOMICS," and allocation of computer resources through "SNIC" and "UNICC" are gratefully acknowledged.

*Present address: Institute of Physics, Tampere University of Technology, 33720 Tampere, Finland.

[1] J. Repp, G. Meyer, F. E. Olsson, and M. Persson, Science 305, 493 (2004).

[2] A. J. Heinrich, J. A. Gupta, C. P. Lutz, and D. M. Eigler, Science 306, 466 (2004).

[3] X.H. Qiu, G. V. Nazin, and W. Ho, Phys. Rev. Lett. 92, 206102 (2004).

[4] S. W. Wu, G. V. Nazin, X. Chen, X. H. Qiu, and W. Ho, Phys. Rev. Lett. 93, 236802 (2004).

[5] J. Repp, G. Meyer, S. M. Stojkovic, A. Gourdon, and C. Joachim, Phys. Rev. Lett. 94, 026803 (2005).

[6] N. Nilius, T. M. Wallis, and W. Ho, Phys. Rev. Lett. 90, 046808 (2003).

[7] Physics of Color Centers, edited by W.B. Fowler (Academic, New York, 1968).

[8] A. L. Shluger and A. M. Stoneham, J. Phys. Condens. Matter 5, 3049 (1993).

[9] V. Zielasek, T. Hildebrandt, and M. Henzler, Phys. Rev. B 62, 2912 (2000).

[10] R. Bennewitz et al., Surf. Sci. 438, 289 (1999).

[11] J. Repp, G. Meyer, and K.-H. Rieder, Phys. Rev. Lett. 92, 036803 (2004).
[12] J. Repp, S. Fölsch, G. Meyer, and K.-H. Rieder, Phys. Rev. Lett. 86, 252 (2001).

[13] As in Ref. [12] we carried out electron-stimulated desorption experiments with the STM in analogous manner to $\mathrm{Cl}$ vacancy creation in bulk $\mathrm{NaCl}$ by an electron beam. It is very difficult to produce just one or only few vacancies using this technique. When approaching the tip to a preadsorbed gold adatom [1] and applying a negative sample voltage in the range of -0.9 to $-1.5 \mathrm{~V}$, an individual $\mathrm{Cl}$ vacancy is formed with a high probability. Even though the mechanism is not understood, the vacancy can be identified from its characteristic STM images as well as in the $d I / d V$ spectra.

[14] G. Kresse and D. Joubert, Phys. Rev. B 59, 1758 (1999).

[15] P. E. Blöchl, Phys. Rev. B 50, 17953 (1994).

[16] G. Kresse and J. Furthmüller, Phys. Rev. B 54, 11169 (1996).

[17] The calculations were carried out in a supercell containing six (four) layers of $\mathrm{Cu}$ with $4 \times 6(6 \times 6) \mathrm{Cu}$ in each layer for $\mathrm{Cu}(311)[\mathrm{Cu}(100)]$, two layers of $\mathrm{NaCl}$, and a vacuum region of $12 \AA$. The relaxation was carried out until residual forces were smaller than $0.05 \mathrm{eV} / \AA$. A dipole correction was applied perpendicular to the surface to correct the effect of surface dipole. Cutoff energy for plane waves was $300 \mathrm{eV}$. The surface Brillouin zone was sampled by a $2 \times 2 k$-point grid.

[18] J. Tersoff and D. R. Hamann, Phys. Rev. Lett. 50, 1998 (1983).

[19] N.D. Lang and A. R. Williams, Phys. Rev. B 16, 2408 (1977).

[20] W. Hebenstreit et al., Surf. Sci. 424, L321 (1999).

[21] F.E. Olsson and M. Persson, Surf. Sci. 540, 172 (2003).

[22] F. E. Olsson, M. Persson, J. Repp, and G. Meyer, Phys. Rev. B 71, 075419 (2005).

[23] The $\mathrm{Cl}$ vacancies in $\mathrm{NaCl}$ films on the $\mathrm{Cu}(100)$ substrate have the same appearance in STM images as for the $\mathrm{Cu}(111)$ substrate.

[24] F. E. Olsson et al., Phys. Rev. Lett. 93, 206803 (2004).

[25] In order to minimize the influence of a nonuniform tip density of states, the ISL spectra are an average of seven spectra acquired with different tip-apex geometries.

[26] J. Kliewer et al., Science 288, 1399 (2000).

[27] J. W. Gadzuk, Phys. Rev. B 44, 13466 (1991).

[28] N. S. Wingreen, K. W. Jacobsen, and J. W. Wilkins, Phys. Rev. B 40, 11834 (1989).

[29] J. D. Dow and D. Redfield, Phys. Rev. B 5, 594 (1972).

[30] The unoccupied Kohn-Sham state energies in DFT do not reproduce the energy of an excitation that involves an addition of a single electron.

[31] If the coupling to the metal states would give rise to a $0.3 \mathrm{eV}$ broadening of the VS, the states energetically within $0.15 \mathrm{eV}$ of the VS should have a considerable weight at the vacancy site.

[32] R. K. Dawson and D. Pooley, Phys. Status Solidi 35, 95 (1969).

[33] The negative differential conductance is due to a tunneling barrier being modified by the applied voltage and is not intrinsic to the system under study. 